\title{
EVIDENCIA ICNOLÓGICA DE UN DINOSAURIO TERÓPODO GIGANTE EN EL BERRIASIENSE (CRETÁCICO INFERIOR) DE LAURASIA (LAS VILLASECAS, SORIA, ESPAÑA)
}

\author{
José Luis BARCO ${ }^{1,2}$, José Ignacio CANUDO ${ }^{1}$, José \\ Ignacio RUIZ-OMEÑACA ${ }^{1}$ y José Luis RUBIO \\ ${ }^{1}$ Area y Museo de Paleontología de la Universidad de Zaragoza. C/ Pedro Cer- \\ buna, s/n. 50009 Zaragoza. jicanudo@unizar.es; jigruiz@unizar.es \\ ${ }^{2}$ Paleoymás, S.L.L. C/ Ntra. Sra. Del Salz, 4. 50017 Zaragoza \\ jlbarco@paleoymas.com \\ ${ }^{3}$ Area de Ecología. Universidad Autónoma de Madrid. Ctra. Colmenar, km 15, \\ 28049 Madrid; joseluis.rubio@uam.es
}

Barco, J. L., Canudo, J. I., Ruiz-Omeñaca, J. I. \& Rubio, J. L. 2005. Evidencia icnológica de un dinosaurio terópodo gigante en el Berriasiense (Cretácico Inferior) de Laurasia (Las Villasecas, Soria, España). [Ichnological evidence of a giant theropod dinosaur in the Berriasian (Lower Cretaceous) of Laurasia (Las Villasecas, Soria, Spain).] Revista Española de Paleontología, N.E. X, 59-71. ISSN 0213-6937.

\begin{abstract}
Comparing to Upper Jurassic and uppermost Lower Cretaceous all over the world, dinosaur fossil record in the Berriasian is small. Wide continental outcrops with great abundance and diversity of theropod, sauropod, ornithopod and avian tracks, Tithonian-Berriasian in age, are usual in Cameros Basin (central and western areas of the Iberian Range, Spain). Although theropod tracks are very abundant, giant theropods are only represented by an isolated footprint from Las Villasecas (Soria province) and a trackway from Treguajantes (La Rioja province), both lower Berriasian in age. The one from Soria is $69 \mathrm{~cm}$ long, and shows thick toes, great claws impressions, absence of hallux, and a characteristic medial displacement of the toe II. Its morphology is similar to the trackway from Treguajantes, made up of three footprints $64-70 \mathrm{~cm}$ in length. Those tracks are different to the theropod icnogenera of similar age and size. Interdigital angles are different in Megalosauripus and Irenesauripus. Bueckeburgichnus shows a clear hallux impression; thus the Spanish tracks could belong to an undescribed ichnogenus. Although giant theropods are developed in several groups from Middle Jurassic to Upper Cretaceous, allosaurids were the group that occupied that role in the Jurassic-Cretaceous transition. However, there are some disagreements about the morphology of allosaurid footprints and cladistic studies prove that Carcharodontosauridae appeared in the Upper Jurassic. So, it is possible that giant theropod dinosaurs belonging to Allosauroidea were present in the Berriasian ecosystems of the Iberian Peninsula, and were the producers of these footprints.
\end{abstract}

Key words: giant theropod, allosauroid, footprint, Berriasian, Cameros Basin, Laurasia, Soria, Spain.

\section{RESUMEN}

El registro mundial de dinosaurios en el Berriasiense es pequeño en comparación con el del Jurásico Superior y el Cretácico Inferior más alto. En la cuenca de Cameros (situada en la Cordillera Ibérica Central y Occidental, España) existen un gran número de yacimientos de edad Titónico-Berriasiense, con gran abundancia y diversidad de icnitas de saurópodos, terópodos, ornitópodos y aves. Las icnitas de dinosaurios terópodos son muy abundantes, sin embargo únicamente la icnita aislada de Las Villasecas (Soria) y un rastro de Treguajantes (La Rioja), ambos registros de edad Berriasiense inferior, pueden asignarse a un terópodo gigante. La icnita soriana mide $69 \mathrm{~cm}$ de longitud y muestra gruesos dedos, impresiones de las uñas muy marcadas, sin impresión de hallux y un característico desplazamiento medial del dedo II. Esa morfología es similar a la del rastro de Treguajantes, compuesto de tres icnitas de entre 64 y $70 \mathrm{~cm}$ de longitud. Estas icnitas son diferentes de los icnogéneros de terópodo de edad y talla similar. Los ángulos interdigitales son diferentes en Megalosauripus e Irenesauripus. Bueckeburgichnus muestra una evidente impresión del hallux, además de otras diferencias morfológicas. Así la icnita española podría pertenecer a un icnogénero aún sin describir. Aunque el gigantismo se ha desarrollado 
en diversos grupos de terópodos desde el Jurásico Medio hasta el Cretácico Superior, los alosáuridos fueron los que ocuparon ese papel en el tránsito Jurásico-Cretácico. Sin embargo existe controversia sobre las icnitas producidas por este grupo, lo que unido a la prueba cladística de la diversificación de los carcarodontosáuridos en el Jurásico Superior, conduce a identificar al posible productor de las icnitas megaterópodas de Cameros como un alosauroideo indeterminado.

Palabras clave: terópodo gigante, alosauroideo, icnita, Berriasiense, Cuenca de Cameros, Laurasia, Soria, España.

\section{INTRODUCCIÓN}

Los terópodos gigantes o megaterópodos se encuentran entre los dinosaurios que ejercen mayor poder de atracción a investigadores y aficionados. El más claro ejemplo es Tyrannosaurus rex Osborn, 1905, que además de ser el más conocido popularmente, es también uno de los dinosaurios que mayor literatura científica ha generado. Sin embargo, los restos fósiles de megaterópodos, salvo algunas excepciones, son escasos y fragmentarios y en muchas ocasiones se reducen únicamente a evidencias paleoicnológicas. Éstas indican que su presencia en los ecosistemas continentales mesozoicos fue constante desde el Jurásico Medio hasta el final del Cretácico (Lockley et al., 1998b).

El gigantismo es un proceso cuyo desarrollo se observa en diversos clados de terópodos a lo largo de la historia evolutiva del grupo. Se pueden citar a los "megalosaurios" en el Jurásico Medio-Superior, los alosáuridos en el Jurásico Superior-Cretácico Inferior, los carcarodontosáuridos en el Cretácico Inferior y los tiranosáuridos en el Cretácico Superior (Bakker, 1998; Holtz, 1998; Allain, 2002). Uno de los periodos geológicos sin registro directo de grandes terópodos es el Berriasiense; sin embargo las evidencias paleoicnológicas estudiadas en este trabajo demuestran su presencia en la Península Ibérica y por tanto en esta parte del supercontinente de Laurasia.

La Cuenca de Cameros es uno de los megayacimientos de icnitas más significativos del mundo (Sanz et al., 1985; Platt \& Meyer, 1991; Moratalla-García, 1993; Moratalla \& Sanz, 1997; Casanovas et al., 1997; Sanz et al., 1999; Pérez-Lorente et al., 2001). Sin embargo su estudio es relativamente reciente ya que comenzó en la década de 1970 (CasanovasCladellas \& Santafé-Llopis, 1971), momento en que ya se encontraron las primeras icnitas de terópodos gigantes en el Cretácico Inferior (Casanovas-Cladellas \& Santafé-Llopis, 1974). En esta cuenca afloran sedimentos continentales de finales del Jurásico (Titónico) y del Cretácico Inferior (Berriasiense-Aptiense) donde existen gran cantidad de icnitas de dinosaurios y otros reptiles mesozoicos. Estos yacimientos, junto a los del resto de la Península Ibérica, constituyen un importante patrimonio científico y cultural, por lo que han sido recientemente propuestos a la UNESCO para su calificación como Patrimonio de la Humanidad.

La mayoría de los yacimientos de la provincia de Soria se sitúan en la comarca de Tierras Altas. Geológicamente se ubican en la subcuenca de Cameros Oriental (Mas et al., 1993) y gran parte de ellos se incluyen en el grupo Oncala (Gómez-Fernández \& Meléndez, 1994). En su estudio han participado varios equipos de investigación (Moratalla, 1993; Sanz et al., 1997; Fuentes Vidarte \& Meijide Calvo, 1998; Lockley et al. 1998a; Pascual-Arribas \& Sanz-Pérez, 2000; Rubio de Lucas, 2001), los cuales han identificado icnitas de dinosaurios terópodos, saurópodos y ornitópodos, así como de otros vertebrados como pterosaurios, cocodrilos y tortugas. Son especialmente abundantes las icnitas atribuidas a dinosaurios terópodos de pequeño y mediano tamaño, habiéndose descrito en los yacimientos sorianos varios icnogéneros e icnoespecies ("Filichnites gracilis" Moratalla-García, 1993; Archaeornithipus meijidei Fuentes Vidarte, 1996; Kalohipus bretunensis Fuentes Vidarte \& Meijide Calvo, 1998; y Therangospodus oncalensis Moratalla, 1993 enmend. Lockley, Meyer \& Moratalla, 1998a).

El icnotaxón más abundante en el Grupo Oncala es Therangospodus Lockley, Meyer \& Moratalla, 1998, atribuido a un dinosaurio terópodo, siendo extremadamente escasa la presencia de icnitas atribuidas a grandes ornitópodos. Sin embargo en el resto del Cretácico Inferior de la Cuenca de Cameros (Grupos Urbión y Enciso) la asociación icnológica es distinta: la presencia de Therangospodus no ha sido documentada (Lockley et al., 1998a), mientras que son abundantes las trazas de grandes ornitópodos y las de terópodos de mayor tamaño asignadas a Bueckeburgichnus Kuhn, 1958 (Moratalla-García, 1993; Moratalla \& Sanz, 1997). Aunque Lockley et al. (1998a) restringen la asociación icnológica Megalosauripus-Therangospodus al Kimmeridgiense, no descartan su presencia en el Cretácico Inferior. Sin embargo, existen evidencias en el grupo Oncala de un gran terópodo que producía icnitas distintas a las descritas como Megalosauripus Lessertisseur, 1955 y Bueckeburgichnus (descartando un solapamiento de icnofaunas). Se trata de un rastro y una icnita aislada halladas en la localidad riojana de Treguajantes (Casanovas et $a l ., 1995)$ y una icnita aislada procedente de Las Villasecas (Soria). Aunque ésta última ya había sido figurada con anterioridad (Rubio de Lucas, 2001; Fuentes Vidarte et al., 2001) y brevemente descrita (Barco et al., 2003), se realiza en este trabajo una descripción en detalle, y se discuten sus afinidades con los icnogéneros morfológicamente más cercanos, lo cual permite caracterizar por primera vez la 
presencia de un terópodo gigante en el Berriasiense de la Península Ibérica, que se convierte a su vez en el mayor terópodo y único terópodo gigante, por el momento, del Cretácico basal de Laurasia.

\section{SITUACIÓN Y ANTECEDENTES}

La icnita estudiada en este trabajo se encuentra depositada en el Museo Numantino y se expone actualmente en el Aula Paleontológica de Villar del Río (Soria). Fue hallada junto al kilómetro 30 de la carretera SO-155 en el término de Villaseca Somera, barrio de Las Villasecas (Fig. 1), por lo que se desconoce el nivel estratigráfico exacto de donde proviene. En este punto aflora una alternancia de areniscas calcáreas y margas de la Aloformación Huérteles del Grupo Oncala, datada como Berriasiense inferior (Gómez Fernández \& Meléndez, 1994; Martín-Closas \& Alonso Millán, 1998). Se interpretan como medios lacustres someros salinos con mayor presencia de sedimentación carbonatada, y ocasionales entradas de material terrígeno a través de ca- nales fluviales. Estos medios lacustres sufrían exposición subaérea en los momentos de sequía produciendo medios adecuados para la formación de icnitas (Moratalla \& Sanz, 1997). La litología en que está conservada la icnita es coherente con este contexto geológico. Se trata de una arenisca carbonatada de color gris que formaba parte de un estrato de unos $35 \mathrm{~cm}$ de potencia. Presenta abundantes galerías de bioturbación de relieve completo, sección circular, trazado rectilíneo y sin bifurcar (semejantes a Planolites Nicholson, 1873). Esta bioturbación se encuentra en el muro, aunque aparentemente no afecta a la icnita.

\section{DESCRIPCIÓN}

La icnita de Las Villasecas está conservada en hiporrelieve convexo y representa el relleno de la impresión producida por un pie izquierdo robusto, mesaxónico y tridáctilo, que destaca por su gran tamaño (Fig. 2a). Presenta la impresión de los dedos II, III y IV gruesos y tenuemente almohadillados, con terminaciones ligeramente redondeadas desde las que se proyectan las

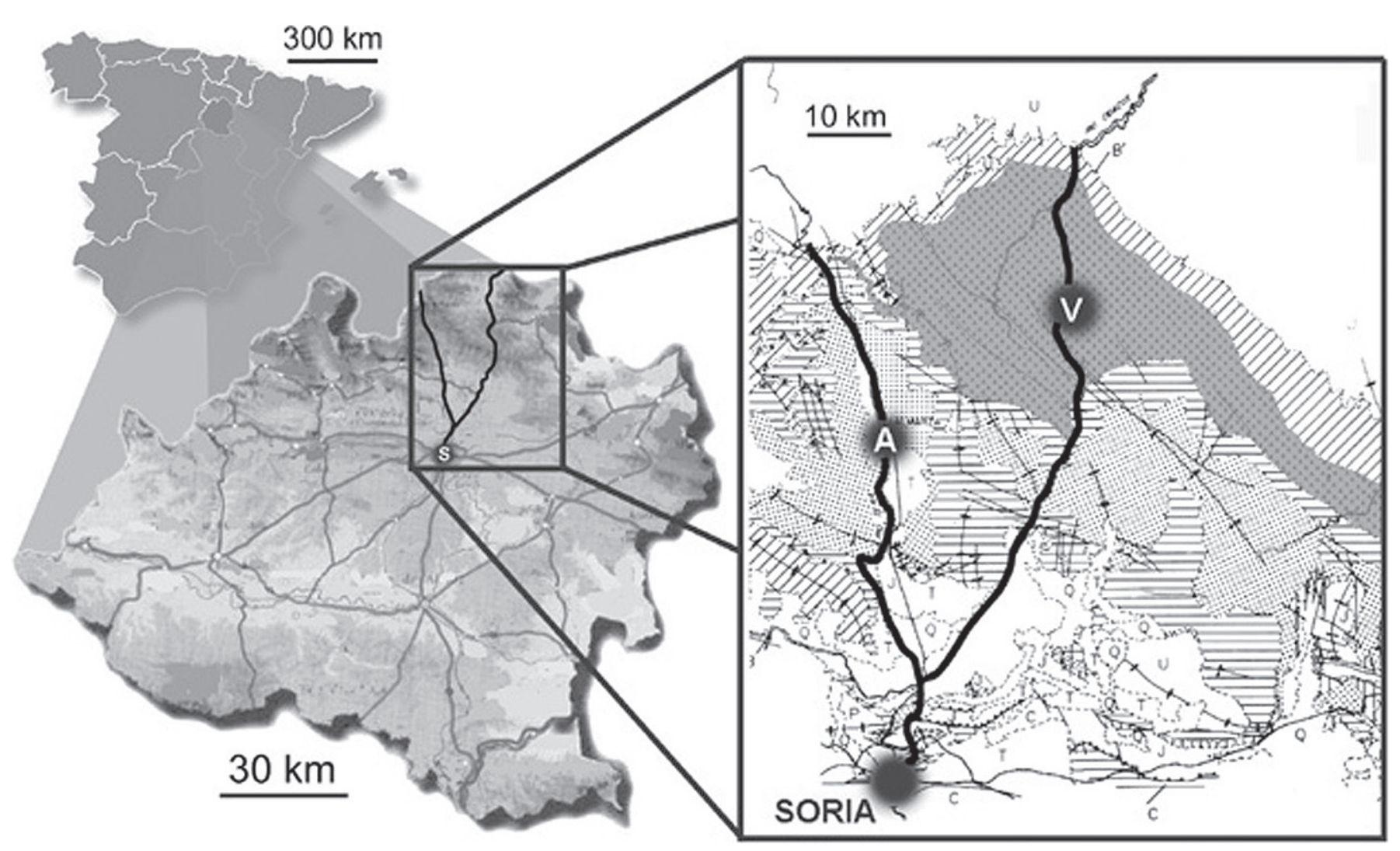

Figura 1. Situación geográfica y geológica de la icnita de Las Villasecas (mapa geológico modificado de Gómez Fernandez \& Meléndez, 1994); sombreada sobre el mapa geológico la Aloformación Huerteles. S: Soria; A: Almarza; V: Situación exacta donde se encontró la icnita de Las Villasecas.

Geographical and geological setting of the Las Villasecas Footprint (geological map modified from Gómez. Fernández \& Meléndez, 1994). Dark area in geological map shows Huerteles Aloformation. S: Soria; A: Almarza; V: Point in wich Las Villasecas footprint was found. 
marcas de gruesas y grandes uñas (entre 5,5 y $7 \mathrm{~cm}$ de longitud). Carece de marca atribuible al hallux (dedo I).

La profundidad máxima de la icnita es de $5,5 \mathrm{~cm}$. La profundidad es más marcada en la zona lateral que en la medial, siendo especialmente evidente en los dedos III y IV. Asimismo las impresiones de los metatarsos son menos profundas que las de los dedos.

La marca de la uña del dedo IV se sitúa en el extremo anterior del dedo, presenta forma cónica, subredondeada en sección y ligeramente curvada hacia el lado medial. La inserción con el resto del dedo es gradual y con forma de letra "V". La zona de inserción de la uña muestra un desplazamiento hacia el lado medial en los tres dedos, ligero en el dedo IV, más marcado en el dedo III, y mucho más claro aun en el dedo II (en este último se sitúa en el área anteromedial). La impresión de la uña del dedo III es subrectangular con una sección cuadrada. El extremo anterior es romo con signos de desgaste y el lado plantar es ligeramente cóncavo, lo que indica que la falange ungueal del productor tenía un amplio aunque poco profundo surco en su parte posterior (Fig. 2b). La unión entre la impresión de la uña del dedo III y el extremo anterior del dedo es neta y recta. La uña del dedo II no se conserva tan nítida, si bien a excepción del mencionado desplazamiento lateromedial y la mayor longitud relativa respecto al dedo, parece ser muy similar a la uña del dedo IV.

Las impresiones de los dedos son distintivamente gruesas: la del dedo IV es más delgada y algo más larga que la del dedo II (Fig. 3), lo que implica que la icnita no es simétrica. El ángulo entre los dedos III-IV, es mayor que el ángulo entre los dedos II-III (Fig. 3). En los tres dedos se observan las marcas de las almohadillas, siendo especialmente claras la IIa, IIIa y la IVa (terminología de Thulborn, 1990), y pudiéndose distinguir que el número de impresiones de almohadillas en los dedos II a IV son 2-3-3 respectivamente, si bien la IIIc está ligeramente enmascarada por las estructuras de refuerzo del talón. Las separaciones entre las almohadillas son suaves y sin apenas constricciones entre ellas, lo cual podría ser indicativo de una deficiente adaptación al suelo de las partes blandas del pie debido a las características del barro. Otra posibilidad para explicar la falta de definición de las almohadillas es, teniendo en cuenta el excelente estado en que se han preservado algunos caracteres de esta icnita, que éstas estuviesen poco definidas en el pie del dinosaurio.

Las impresiones de la zona distal de los metatarsos no son muy profundas, posiblemente debido al desplazamiento del barro tras la realización de la pisada. El desprendimiento del barro se observa también en marcas presentes en los dedos III (con dos de ellas muy evidentes a simple vista) y IV, indicativas de que fragmentos de sustrato quedaron adheridos al pie del dinosaurio. La impresión distal del metatarso IV es grande, subredondeada y corresponde a la parte más posterior de la icnita. La del metatarso II es más pequeña lo que provoca presencia de una escotadura muy habitual en las huellas terópodas, aunque en este caso no esté demasiado marcada. La terminación proximal de la icnita coincide con la impresión de la zona distal del metatarso del dedo $\mathrm{IV}$, sin que existan evidencias de apoyo de ninguna parte más posterior, lo que indica claramente que toda la icnita se debe a una impresión dactilar del pie del dinosaurio.

En el punto de unión de los dedos II y III y, de forma simétrica aunque menos marcada, entre los dedos III y IV, se observan unas estructuras convexas semejantes a almohadillas pero con forma más alargada. Se ha considerado que estas morfolo-
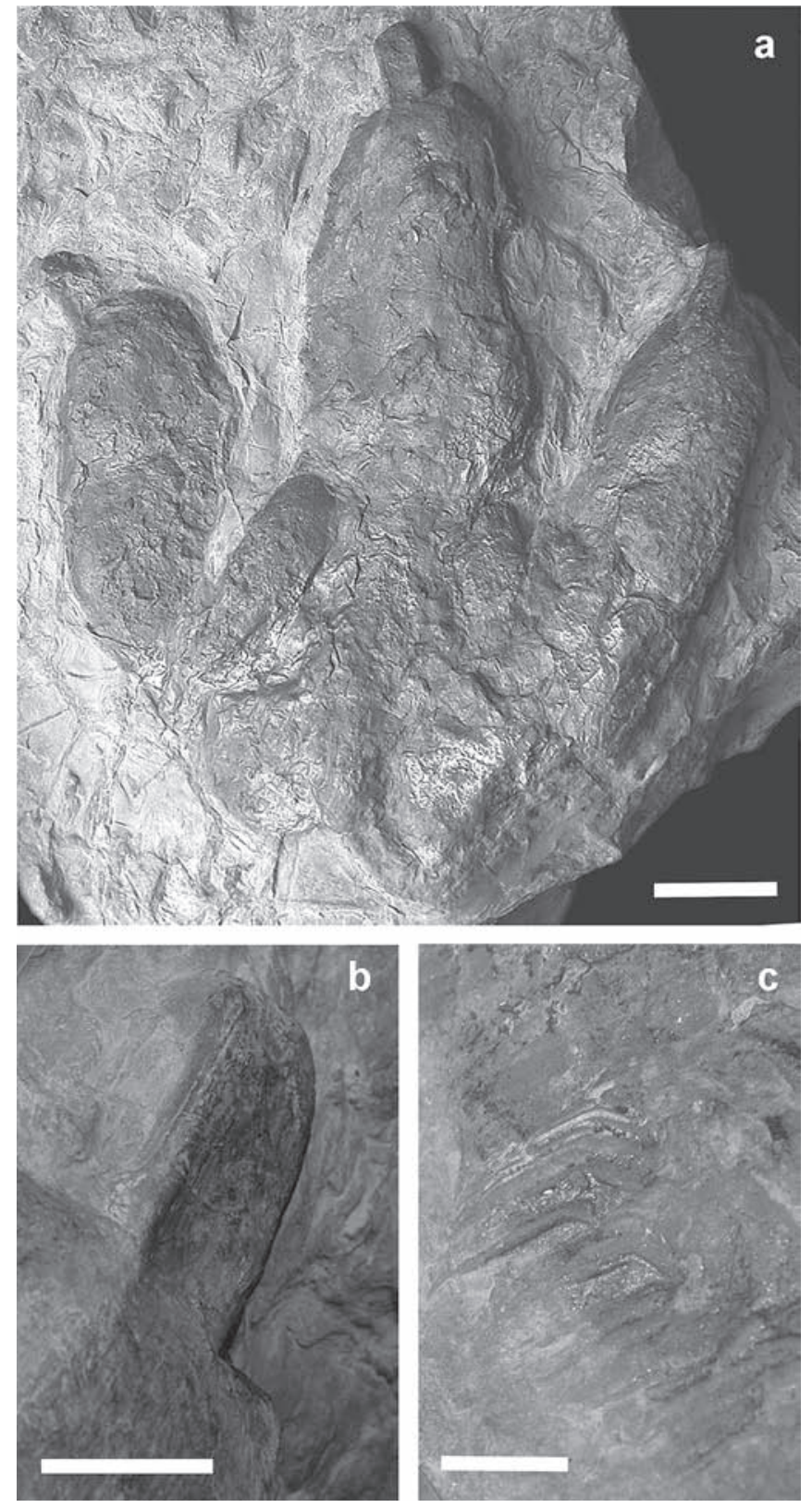

Figura 2. a: la icnita de Las Villasecas (escala: $10 \mathrm{~cm}$ ). b: detalle de la marca de la uña del dedo III (escala: $3 \mathrm{~cm}$ ). c: detalle de marcas de piel (escala: $2 \mathrm{~cm}$ ).

$\boldsymbol{a}$ : Las Villasecas footprint (scale bar: $10 \mathrm{~cm}$ ). $\boldsymbol{b}$ : claw impression of the toe III in detail (scale bar: $3 \mathrm{~cm}$ ). c: skin impressions in detail (sacle bar: $2 \mathrm{~cm}$ ).

gías han sido producidas por estructuras presentes en el pie del dinosaurio (ver discusión), interpretadas como pliegues o almohadillas de "refuerzo".

En toda la icnita aparecen estrías longitudinales, más evidentes en la impresion de la uña del dedo III, en la parte anterior de los dedos y en la zona de los hypex. Se observan también otras más gruesas dispuestas en sentido dorsoventral y ligeramente inclinadas hacia delante, las cuales sólo aparecen en la parte más anterior del dedo III y posiblemente correspondan a impresiones de la piel (Fig. 2c). 


\section{DISCUSIÓN}

La presencia de marcas de arrastre de piel, junto a su buena conservación, indican que la icnita de Las Villasecas es una icnita verdadera y no un subhuella. Además no presenta evidencias de adelgazamiento de los dedos por la caída de barro al interior de la pisada, por lo que refleja con bastante fidelidad la morfología del pie del dinosaurio.

La terminación cuadrangular, aunque con extremos romos y no abruptos, de la uña del dedo central se interpreta como causa de un desgaste continuo producido en vida, más que como atribuible a una rotura.

Esta icnita presenta una novedad como son dos estructuras almohadilladas situadas de manera simétrica en la parte posterior del dedo III. En una observación somera podría parecer que estas marcas han sido producidas por una pisada distinta y por tanto pertenecen a otra icnita. Sin embargo, al realizar un estudio en profundidad, se observa que estas estructuras no han sido afectadas ni afectan a las almohadillas de la icnita megaterópoda (descartando que se trate de una impresión previa o posterior respectivamente), sino que además se adaptan a ellas, por lo que esta morfología sólo puede explicarse como producida por estructuras pertenecientes al pie del dinosaurio. Su interpretación es más problemática, ya que aunque por su morfología indica que podrían ser almohadillas, no se adaptan al patrón metatarsal clásico al no tener ninguna relación con las falanges. Su simetría a ambos lados de la icnita descarta en principio que se trate de una posible patología, siendo más indicativa de una estructura propia del pie que, en el estado de conocimiento actual, puede ser considerada como almohadillas de refuerzo o simples pliegues de la piel.

El pie que produjo la huella de Las Villasecas era funcionalmente tridáctilo y la icnita no es lo suficientemente profunda como para que el hallux alcanzase el suelo dejando su impresión. Un pie funcional tridáctilo más largo que ancho, con falanges ungueales estrechas y agudas, indica que el dinosaurio responsable de la pisada es un terópodo. Otros caracteres que confirman este hecho son la presencia de escotadura en posición posterior a la impresión del dedo II, y la impresión de la uña del dedo II orientada hacia la parte medial de la icnita (Thulborn, 1990; Pittman, 1992; Moratalla-García, 1993; Lockley, 1998).

El tamaño de la icnita de Las Villasecas indica que fue producida por un megaterópodo. Tradicionalmente, las icnitas de grandes terópodos, denominadas de manera informal como carnosaurias, se han diferenciado de las llamadas celurosaurias por el tamaño, habiéndose establecido el límite entre unas y otras en los $25 \mathrm{~cm}$ de longitud (Thulborn, 1990). Este mismo autor cita una icnita de 80 cm de longitud como la más grande atribuida a un carnosaurio, si bien no especifica al dinosaurio responsable. Otras icnitas atribuidas a un terópodo (Gigantosauropus

\begin{tabular}{|l|r|}
\hline L & 69 \\
\hline A & 56 \\
\hline LII & 33,6 \\
\hline LIII & 50 \\
\hline LIV & 47 \\
\hline AII & 15 \\
\hline AIII & 18 \\
\hline AIV & 12,8 \\
\hline II^III & 12 \\
\hline III^IV & 21 \\
\hline LgII & 7 \\
\hline LgIII & 6,5 \\
\hline LgIV & 5,5 \\
\hline
\end{tabular}

A

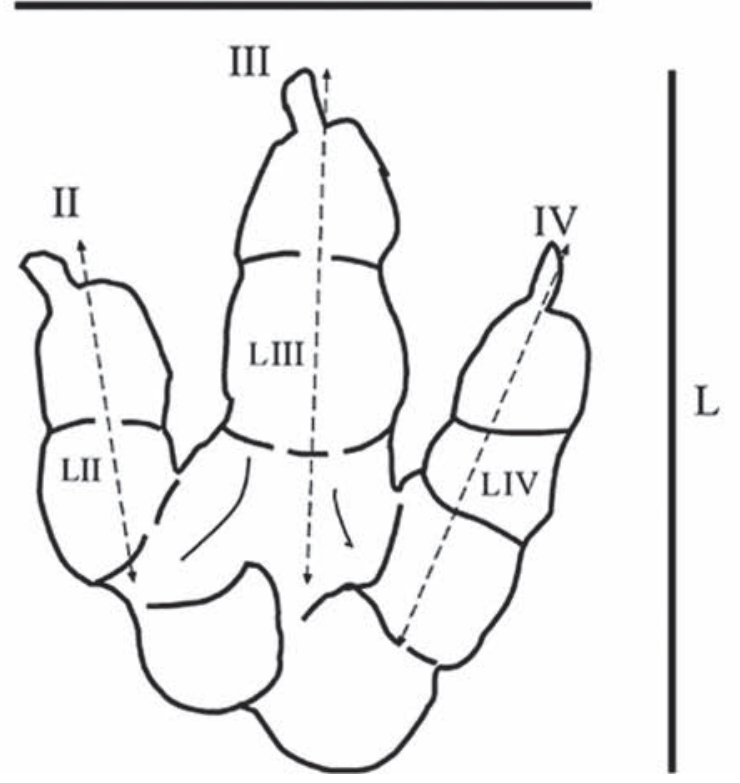

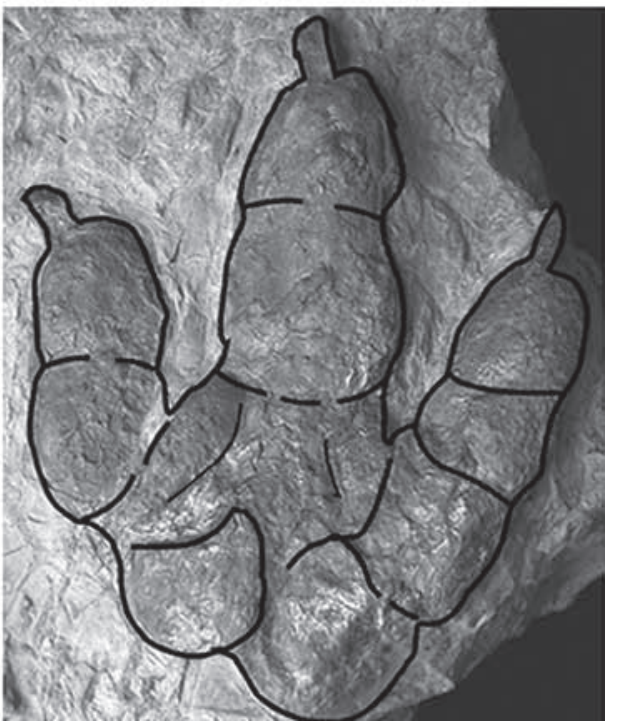

Figura 3. Medidas de la icnita de Las Villasecas en cm. L: longitud; A: anchura; LII: longitud del dedo II; LIII: longitud del dedo II; LIV: longitud del dedo IV; II^III: ángulo entre los dedos II y III; III^IV: ángulo entre los dedos III y IV; LgII: longitud de la uña del dedo II; LgIII: longitud de la uña del dedo III; LgIV: longitud de la uña del dedo IV.

Las Villasecas footprint measurements in cm. L: length; A: width; LII: length of toe II; LIII: length of toe II; LIV: length of toe IV; II^III: angle between toes II and III; III^IV: angle between toes III and IV; LgII: length of claw impression of the toe II; LgIII: length of claw impression of the toe III; LgIV: length of claw impression of the toe IV. 
Mensink \& Mertmann, 1984), de $150 \mathrm{~cm}$ de longitud, son en realidad de saurópodo (Thulborn, 1990; Lockley \& Hunt, 1994; Lires et al., 2001).

En este trabajo se emplea el término megaterópodo para designar a los dinosaurios cuya altura hasta el acetábulo, o longitud funcional del miembro trasero, sea igual o superior a los $250 \mathrm{~cm}$, y que por tanto produciría, según la fórmula de Alexander (1976), icnitas con una impresión plantar de $52 \mathrm{~cm}$ de longitud o más. Según estos parámetros, la altura al acetábulo del dinosaurio responsable de la icnita de Las Villasecas era de $328 \mathrm{~cm}$ (la altura total a la parte superior de la cadera superaría los $3,5 \mathrm{~m}$ ) y dentro de los megaterópodos sólo sería superado por $\mathrm{Me}$ galosauripus del Jurásico Superior de Portugal (Lockley et al., 1998b) y Tyrannosauripus Lockley \& Hunt, 1994, del Cretácico Superior de EEUU, que alcanzarían aproximadamente unos 3,8 $\mathrm{m}$ de altura hasta el acetábulo. Pese a que la totalidad de la pisada de Tyrannosauripus mide $86 \mathrm{~cm}$, ésta muestra clara evidencias de la impresión de una buena parte de la zona distal del metatarso, por lo que para éste calculo se ha utilizado la longitud del pie digitigrado, que estimamos entre 74 y $76 \mathrm{~cm}$.

En la formación Oncala de la Cuenca de Cameros sólo se han citado icnitas morfológicamente semejantes a la de Las Villasecas en la localidad riojana de Treguajantes (Casanovas et al., 1995). Se trata de un rastro (Fig. 4) y una icnita aislada, sin marca de hallux, el dedo II más corto y ancho que el IV y una relación semejante entre longitud y anchura (ver tabla en Casanovas et al., 1995) a la icnita de Las Villasecas, lo que unido a que se sitúa en los mismos niveles estratigráficos, nos lleva a atribuirlas a un mismo tipo de terópodo.

Tradicionalmente en paleoicnología, muchas de las icnitas de grandes terópodos han sido agrupadas como Megalosauripus, Tyrannosauripus y términos similares. Recientes trabajos (Lockley \& Hunt, 1994; Lockley, 1998; Lockley et al., 1996, 1998b) han contribuido notablemente a clarificar la sistemática de las grandes icnitas de terópodos poniendo de manifiesto las diferencias entre las halladas en el Jurásico y en el Cretácico y evidenciando icnogéneros y morfotipos distintos (Moratalla-García,1993; Wright et al.,1998; Lockley, 1998) algunos de los cuales será necesario describir.

El único registro Berriasiense de icnitas atribuidas a terópodos gigantes es el icnogénero Bueckeburgichnus (Fig. 5), descrito en Alemania por Abel (1935) y recientemente enmendado por Lockley (2000). Según este último autor es un morfotipo claramente distinguible por tener bien marcada la impresión de las almohadillas del dedo II y la del dedo IV más estrecha y ligeramente segmentada. Este icnogénero difiere de la icnita de Las Villasecas en que sus dedos II y III son mucho más delgados, el dedo III se hace gradualmente más delgado hacia el extremo distal presentando una ligera curvatura hacia el lado lateral y los ángulos interdigitales son mucho mayores. El icnogénero alemán muestra además la presencia de hallux, aunque, como ya apunta Lockley (2000), éste es un carácter en el que influyen diversas variables como la profundidad y conservación de la impresión y su carácter diagnóstico es, cuando menos, dudoso.

En la Cuenca de Cameros se han citado icnitas de dedos anchos y robustos, con una relación longitud / anchura entre 0,95 y 1,2 y con presencia de hallux, atribuidas a Bueckeburgichnus (ver Moratalla \& Sanz, 1997). Los ejemplos más claros de la presencia de este icnogénero se encuentran en el yacimiento aptiense de Los Cayos, aunque en este caso rara vez superan los $45 \mathrm{~cm}$ de longitud (Moratalla-García, 1993). Sin embargo las icnitas más grandes descritas en la Cuenca de Cameros y en España son las de Treguajantes (Casanovas et al., 1995; Pérez-Lorente, 2004), una de las cuales alcanza los $70 \mathrm{~cm}$ y que como se discute anteriormente presentan, junto a la icnita estudiada en este trabajo, caracteres distintos de Bueckeburgichnus, lo que implica que al menos hay dos icnotaxones de grandes terópodos en el Cretácico Inferior de la Cuenca de Cameros.

Los icnogéneros de terópodos más cercanos en el tiempo a las icnitas de Las Villasecas y Treguajantes son
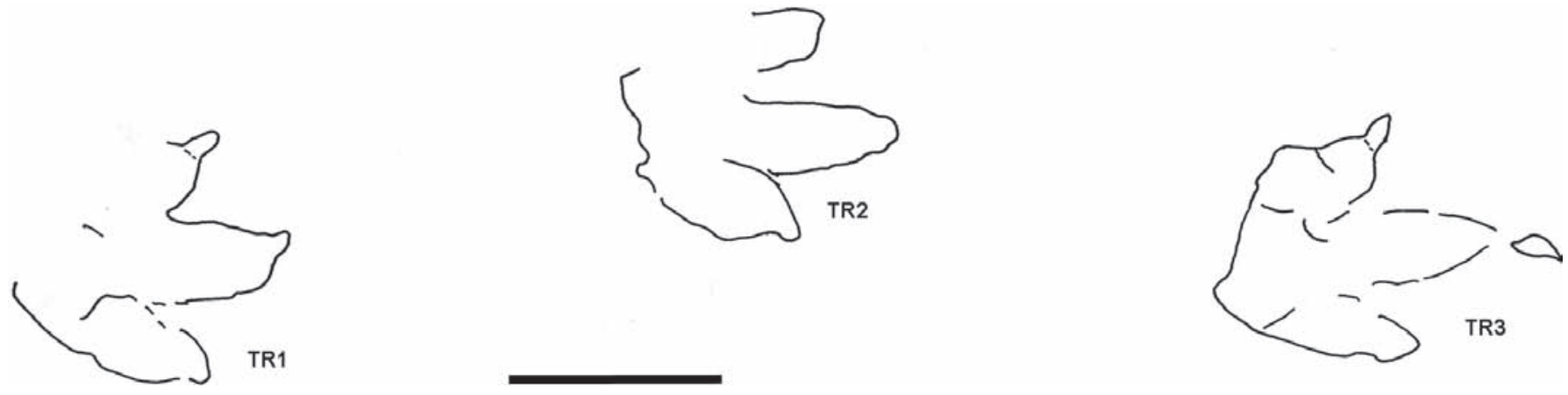

Figura 4. Rastro TR1 del yacimiento de Treguajantes (modificado de Casanovas et al., 1995). Escala: $50 \mathrm{~cm}$. Trackway TR1 from Treguajantes site (modified from Casanovas et al., 1995). Scale bar: $50 \mathrm{~cm}$. 


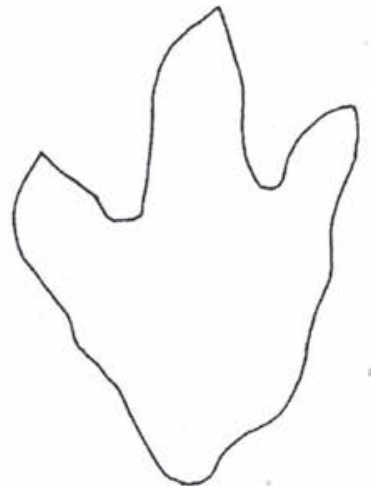

Megalosauripus

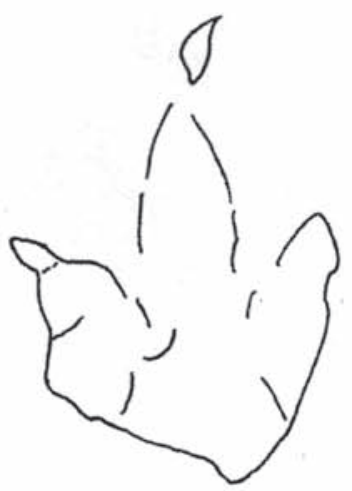

Treguajantes

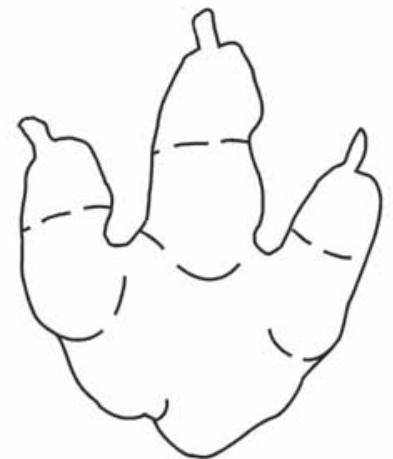

Las Villasecas

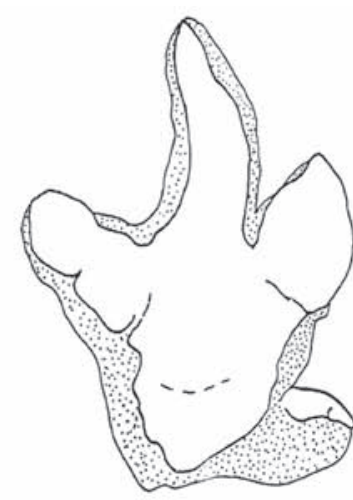

Bueckeburgichnus

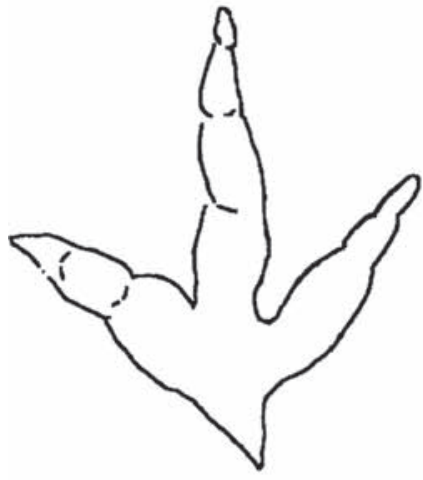

Irenesauripus

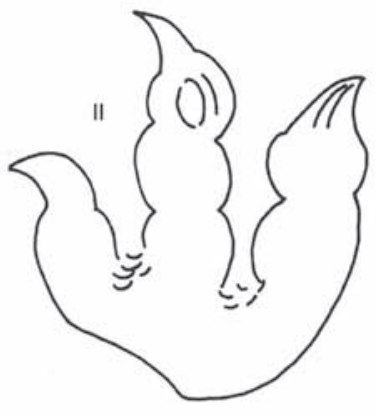

Abelichnus

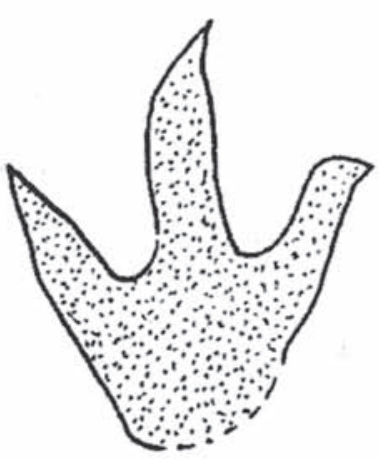

"Eubrontes" glenrosensis

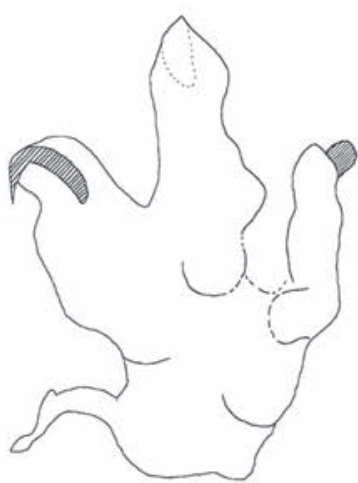

Tyrannosauripus

Figura 5. Icnitas de megaterópodos. Tomadas de: Lockley et al., 1998b (Megalosauripus, Irenesauripus, Tyrannosauripus, "Eubrontes" glenrosensis, Abelichnus); Casanovas et al., 1995 (Treguajantes); Lockley, 2000 (Bueckeburgichnus).

Megatheropod footprints. From: Lockley et al., 1998 (Megalosauripus, Irenesauripus, Tyrannosauripus, "Eubrontes" glenrosensis, Abelichnus); Casanovas et al., 1995 (Treguajantes); Lockley, 2000 (Bueckeburgichnus).

Megalosauripus (Fig. 5) e Hispanosauripus Mensink \& Mertmann, 1984. En el icnogénero Megalosauripus se incluyen las mayores icnitas de terópodos jurásicos (Lockley et al., 1998b). Las más grandes $(77 \mathrm{~cm})$, actualmente desaparecidas debido al desprendimiento del estrato donde se encontraban, han sido citadas en Zambujal, Portugal (Santos et al., 1995). Uno de los mejores ejemplos de este icnogénero son las identificadas como Megalosauripus uzbekistanicus (Gabuniya \& Kurbatov, 1982), del Oxfordiense superior-Kimmeridgiense inferior de Uzbekistan (Lockley et al., 1996, 1998b). Son también de gran tamaño $(72 \mathrm{~cm})$, tridáctilas, alargadas (relación longitud / anchura de la icnita, excluyendo el metatarso, varía entre 1,35 y 1,64), presentan unas impresiones de almohadillas bien marcadas, con la formula típica para los terópodos $(2,3$ y 4 correspondiente a los dedos II, III y IV) y un talón alargado (relación de la longitud del pie / longitud del dedo III es 1,45-1,85). Los rastros de Megalosauripus se caracteri- zan por ser muy variables, de estrechos a moderadamente anchos, y con un ángulo de paso que oscila entre $125^{\circ} \mathrm{y}$ $175^{\circ}$ (Lockley et al., 1998b). Pese a carecer también de impresión del hallux, Megalosauripus se diferencia de la icnita de Las Villasecas en que sus ángulos interdigitales son ligeramente superiores, al igual que la relación entre la medida de la impresión del talón y la del dedo III. La relación entre longitud y anchura es también ligeramente superior en Megalosauripus. Finalmente todas las icnitas atribuidas a Megalosauripus, muestran claramente que tanto las marcas de sus almohadillas como las impresiones de sus dedos son más delgadas que las icnita soriana.

Hispanosauripus únicamente ha sido hallado en el Jurásico Superior de Asturias, donde fue definido por Mensink \& Mertmann (1984), y posteriormente citado por Valenzuela et al. (1986, 1988). Este icnogénero está constituido por icnitas asimétricas, que conservan las impresiones de las almohadillas. La longitud del dedo III es cerca del $60 \%$ 
de la longitud del pie (lo que implica una relación entre longitud total y longitud del dedo de 1,66) y el ángulo de de paso oscila entre $125-175^{\circ}$. En base a estos caracteres es difícil diferenciar a Hispanosauripus de Megalosauripus (Lockley et al., 1998b), si bien es posible descartar cualquier similitud con la icnita de Las Villasecas. Posteriores trabajos han considerado a Hispanosauripus como nomen nudum (Lires et al., 2001).

En niveles más modernos del Cretácico se han descrito varios icnogéneros de grandes terópodos. Así en el Cretácico Inferior (Aptiense-Albiense) de Canadá se ha descrito Irenesauripus Sternberg, 1932 (Sternberg, 1932; Lockley, 1998), taxón cuyas icnitas presentan marcadas diferencias con la de Las Villasecas al ser más gráciles, con los dedos finos, adelgazados gradualmente y con un ángulo interdigital grande que oscila siempre alrededor de $100^{\circ}$ (Lockley, 1998) (Fig. 5). En niveles estratigráficos similares, en el Albiense de Texas (EEUU), están descritas las icnitas de un gran terópodo sin impresiones de las almohadillas, denominadas como Eubrontes glenrosensis Shuler, 1935 aunque esta asignación es inadecuada (Lockley et al., 1998b), siendo necesario asignarlas a un nuevo icnogénero. Las impresiones de los dedos son más estrechas que las de la icnita de Las Villasecas, y aunque esto podría ser resultado del desplazamiento de barro hacía el hueco de la pisada, el taxón tejano presenta los dedos II y IV de un tamaño similar y no más ancho el II como en Las Villasecas (Fig. 5).

En el tránsito entre el Cretácico Inferior y el Superior se ha citado Abelichnus Calvo, 1991 (Fig. 5), icnogénero con icnitas de hasta $50 \mathrm{~cm}$ descrito en el Cenomaniense de Neuquén, Argentina (Calvo, 1991, 1999; Meyer, 2000). Son tridáctilas sin impresión del hallux y con grandes y gruesas impresiones de los dedos. Las uñas son anchas y ocupan por completo el extremo anterior de la impresión del dedo, especialmente en el dedo IV. La impresión de los dedos ocupa la mayor parte de la impresión de la icnita. Estos caracteres lo diferencian claramente de la icnita de Las Villasecas.

En el Cretácico Superior el único icnogénero de megaterópodo que se considera válido es Tyrannosauripus (Fig. 5). Está descrito con una icnita aislada de la Formación Ratón (Maastrichtiense) en Nuevo México, EEUU (Lockley \& Hunt, 1994), y es la icnita de terópodo más grande descrita $(86 \mathrm{~cm})$. El holotipo de Tyrannosauripus pillmorei Lockley \& Hunt, 1994, a diferencia de la icnita de Las Villasecas, presenta un hallux bien desarrollado que se proyecta medialmente, es más asimétrica, la posición de las uñas es más variable y carece de impresión de las almohadillas. El único rasgo semejante entre ambos registros es la impresión del dedo IV, significativamente más delgada que la del dedo II (Lockley, 1998), pese a lo cual consideramos que se trata de distintos taxones. Lockley (1998) dice que en el holotipo de Tyrannosauripus no se observa bien la impresión de las almohadillas, apuntando como razonable que no se marquen por los refuerzos ( $p a d$ ) de carne que tendría el pie de un animal de más de cinco toneladas.

Por todo ello, la icnita de Las Villasecas no puede asignarse a ninguno de los icnogéneros de megaterópodo conocidos, por lo que posiblemente pertenece a un icnogénero sin describir.

\section{DINOSAURIOS DEL BERRIASIENSE ¿CÚAL ES EL PRODUCTOR?}

El Berriasiense es uno de los periodos con menor registro fósil de dinosaurios a nivel mundial. En Europa sólo se han descrito tres especies de terópodos berriasienses: "Prodeinodon" dunkeri (Dames, 1884) en Alemania, Nuthetes destructor Owen, 1854 en Inglaterra y Dromaeosauroides bornholmensis Christiansen \& Bonde, 2003 en Dinamarca. El primero es un manirraptor grande (Ruiz-Omeñaca \& Canudo, 2003); el diente holotipo tiene $22 \mathrm{~mm}$ de longitud mesiodistal, lo que puede indicar una longitud total de aproximadamente 8,5 m comparando con el holotipo de Saurornithoides mongoliensis Osborn, 1924 (Osborn, 1924; Paul, 1988), y una altura al acetábulo de alrededor de 2,2 m (extrapolada de la reconstrucción de este mismo género figurada por Paul, 1988). Los otros dos taxones son dromeosáuridos de pequeño tamaño (Milner, 2002; Bonde $\&$ Christiansen, 2003). Como ha quedado patente anteriormente, la icnita de Las Villasecas y el rastro de Treguajantes pertenecen a un megaterópodo, por lo que no pudieron ser producidos por ninguno de estos tres taxones.

Se ha citado también Aristosuchus sp. en el Berriasiense de Rumania (Jurcsák, 1982; Jurcsák \& Popa, 1983; Benton et al., 1997), los restos, dos vértebras, son muy escasos para permitir una asignación a este género de compsognátido (Naish, 2002) cuyo holotipo es un sacro y dos pubis asociados del Barremiense de Inglaterra, pertenecientes a un animal con una longitud aproximada de $2 \mathrm{~m}$ (Naish et al., 2001; Naish, 2002). En cualquier caso, estas vértebras, junto con dos falanges ungueales (Theropoda indet.; Grigorescu, 2003), representarían a un terópodo de sólo 2-3 m de longitud (Benton et al., 1997: 283).

Otros posibles terópodos presentes en el Berrasiense europeo son un alosauroideo, representado por dientes aislados de tamaño grande del Purbeck de Inglaterra (Milner, 2002), aunque no tienen caracteres diagnósticos y su asignación a este grupo es muy discutible, y un Maniraptora de tamaño grande, representado por un metatarso, también del Purbeck inglés (Milner, 2002).

La morfología y las proporciones de las falanges del pie varían dentro de los distintos clados de grandes terópodos. En consecuencia no es posible asignar inequívocamente unas icnitas determinadas a un taxón en concreto. Sin em- 
bargo, la presencia de restos directos de terópodos en áreas geográfica y temporalmente similares, permiten proponer al candidato más adecuado (Thurlborn, 1990). Uno de los más claros ejemplos es Tyrannosauripus y Tyrannosaurus Osborn, 1905, cuya relación proviene de la presencia de ambos en las mismos niveles estratigráficos (Lockley \& Hunt, 1994). Un caso similar se da en los niveles donde aparece Abelichnus, en los que el único gran terópodo encontrado es Giganotosaurus Coria \& Salgado, 1995, por lo que las icnitas han sido relacionadas con ese género (Calvo, 1999; véase también Meyer, 2000), pasando a ser las únicas asignadas a un carcarodontosáurido. Finalmente también se ha relacionado algunas grandes icnitas del Cretácico Inferior de Texas con el alosáurido Acrocanthosaurus Stovall \& Langston, 1950 (Langston, 1974; Pittman, 1989; Farlow, 2001). Mediante un repaso a la filogenia de los grandes terópodos y sus ocurrencias temporales y paleogeográficas, se realiza a continuación una aproximación al posible productor de la icnita se Las Villasecas.

El gigantismo se ha dado en diferentes grupos de terópodos: allosáuridos (Acrocanthosaurus, Allosaurus=Epanterias, Saurophaganax; Smith, 1998; Chure, 2000; Currie \& Carpenter, 2000) en el Jurásico Superior y Cretácico Inferior, carcarodontosáuridos (Carcharodontosaurus, Giganotosaurus; Coria \& Salgado, 1995; Sereno et al., 1996; Calvo \& Coria, 1998) y espinosáuridos (Spinosaurus; Paul, 1988; Milner, 1997) en el límite Cretácico Inferior-Cretácico Superior, y tiranosáuridos (Tarbosaurus, Tyrannosaurus; Hurum \& Sabath, 2003; Currie et al., 2003; Currie, 2003) en el Cretácico Superior.

Uno de los análisis más completos a la hora de clarificar las relaciones de los grandes terópodos es el de Allain (2002), quien entre otras divisiones propone la de Spinosauroidea en dos familias: Spinosauridae y Megalosauridae. Este clado de tetanuros basales se diversificaron en el Jurásico Inferior (Holtz, 1998; Allain, 2002). Los espinosáuridos son desconocidos en el Jurásico y en la parte baja del Cretácico Inferior, sin embargo es una familia bien conocida a partir del Barremiense en Gondwana y Laurasia (Kellner \& Campos, 1996; Russell, 1996; Charig \& Milner, 1997; Sereno et al., 1998; Ruiz-Omeñaca et al., 1998; Buffetaut \& Ouaja, 2002; Sues et al., 2002), siendo los del Hauteriviense superior de la Península Ibérica los niveles más antiguos en los que se han encontrado por el momento (Ruiz-Omeñaca \& Canudo, 2001). No se han asignado icnogéneros a esta familia que permitan una comparación con la icnita de Las Villasecas, pero el rango temporal mencionado, permite descartar a los espinosaúridos como productores de la icnita estudiada.

La familia Megalosauridae, según Allain (2002), se encuentra bien representada en el Jurásico de Laurasia: Jurásico Medio de Europa (Megalosaurinae) y Jurásico Superior de Norteamérica (Torvosaurinae). La única cita de Gondwana es de niveles del Cretácico Inferior del norte de África (Afrovenator; Sereno et al., 1996). Los megalosáuridos se diversificaron en el Jurásico, por lo que la relación que proponen Lockley et al. (1998b) en su diagnosis enmendada de Megalosauripus, que indica que el nombre implica relación con dinosaurios similares a Megalosaurus y sus parientes, puede ser adecuada. Esto implica que, ni por posición estratigráfica ni por afinidades con Megalosauripus, la icnita de Las Villasecas puede asignarse a un megalosáurido.

Aunque hay tiranosauroideos en el Jurásico Superior de Portugal (Aviatyrannis jurassica Rauhut, 2003) y Cretácico Inferior de Inglaterra (Eotyrannus lengi Hutt, Naish, Martill, Barker \& Newbery, 2001), su tamaño es pequeño-mediano (Hutt et al., 2001; Naish et al., 2001; Rauhut, 2003), y sólo alcanzaron tamaños gigantes en el Cretácico Superior (Rauhut, 2003: 908), por lo que no pudieron ser los productores de la icnita de Las Villasecas.

Allosauroidea es otro de los clados con presencia de gigantismo. Las familias de estos grandes neotetanuros tienen un marcado provincialismo (Allain, 2002): Sinraptoridae en el Jurásico Superior de Asia (Currie \& Zhao, 1993), Carcharodontosauridae en el Cretácico Inferior de Gondwana (Coria \& Salgado, 1995; Russell, 1996; Sereno et al., 1996; Calvo \& Coria, 1998) y Allosauridae en el Jurásico Superior y Cretácico Inferior de Europa (PérezMoreno et al., 1999; Hutt et al., 1996; Naish et al., 2001) y Norteamérica (Madsen, 1976; Harris, 1998; Smith, 1998; Currie \& Carpenter, 2000; Chure, 2000). La cita de dientes aislados en el Purbeck inglés (Berriasiense) similares a los de Allosaurus (Milner, 2002), confirma la presencia de alosauroideos en el Berriasiense de Europa y a su vez la convierte en la única familia de megaterópodos reconocida hasta el momento en esta edad. Por ello, en una primera aproximación, se puede suponer que el productor de la icnita de Villasecas es un representante de Allosauridae, si bien esto genera algunas dudas. Las icnitas de megaterópodo del Albiense de Texas (conocidas como "Eubrontes" glenrosensis) han sido atribuidas al alosáurido Acrocanthosaurus (Langston, 1974; Pitman, 1989; Farlow, 2001). Estas icnitas, como ya se ha discutido, son diferentes a las icnitas de megaterópodos de Cameros, si bien los dedos en ambas son gruesos. Sin embargo McRea et al. (2002) proponen que el icnogénero Irenesauripus con dedos finos, también había sido producido por un terópodo de la familia Allosauridae. Por otro lado, aunque se desconoce cuando pudieron alcanzar el gigantismo, los estudios cladísticos apuntan a que la familia Carcharodontosauridae pudo diversificarse ya en el Jurásico Superior (Allain, 2002) y la forma del pie de los terópodos de esta familia es coherente con la forma de las icnitas de Las Villasecas y Treguajantes. Este hecho, junto a la falta de consenso en torno a las icnitas de la familia Allosauridae, nos lleva a proponer como posible productor de las icnitas de me- 
gaterópodos estudiadas en este trabajo a un alosauroideo indeterminado.

\section{CONCLUSIONES}

La icnita de Las Villasecas, en Soria, junto a las Treguajantes en la Rioja, constituyen la primera evidencia de un megaterópodo en el Berriasiense de la Península Ibérica, siendo además evidencia del mayor terópodo y único megaterópodo, por el momento, del Cretácico basal de Laurasia. Se trata también de uno de más grandes registrados en el mundo, ya que los $3,28 \mathrm{~m}$ de altura a la cadera que presentaría el organismo productor sólo serían superados según las evidencias icnológicas, por los productores de Megalosauripus del Jurásico Superior de Portugal y de Tyrannosauripus del Cretácico Superior de EEUU.

El contexto cronológico y el estado actual de conocimientos sobre los dinosaurios terópodos, parece indicar que la icnita de Las Villasecas pudo ser producida por un neotetanuro alosauroideo de gran tamaño. Aunque los alosáuridos son los únicos megaterópodos citados en el Cretácico Inferior de Europa y Norteamérica, no existe consenso sobre la morfología de las icnitas producidas por esta familia y algunas de las que les han sido atribuidas son más gráciles que las españolas. Debido a que otras familias de terópodos gigantes pertenecientes al clado Allosauroidea, como Carcharodontosauridae, ya se habían diversificado a finales del Jurásico, se considera que el productor de la icnita estudiada debió ser un representante del clado Allosauroidea.

\section{AGRADECIMIENTOS}

Los autores agradecen a la Junta de Castilla y León y al Museo Numantino las facilidades ofrecidas para el estudio. El Grupo de Investigación en Dinosaurios de la Universidad de Zaragoza esta financiado por el Ministerio de Ciencia y Tecnología (proyecto VECOBA, ref. BTE 2001-1746). La empresa Paleoymás financia parte de las investigaciones de J.L. Barco.

\section{BIBLIOGRAFÍA}

Abel, O. 1935. Vorzeitliche Lebensspuren. Ed. Gustav Fischer, Jena, 644 pp.

Alexander, R. M. 1976. Estimates of speeds of dinosaurs. Nature, 261, 129-130.

Allain, R. 2002. Discovery of megalosaur (Dinosauria, Theropoda) in the middle Bathonian of Normandy (France) and its implications for the phylogeny of basal tetanurae. Journal of Vertebrate Paleontology, 22, 548-563.

Bakker, R.T. 1998. Brontosaur killers: Late Jurassic allosaurids as sabre-tooth cat analogues. In: Aspects of Theropod
Paleobiology (Eds. B. P. Pérez Moreno, T. Holtz, Jr., J.L. Sanz \& J. Moratalla.). Gaia, 15, 145-158.

Barco, J. L., Canudo, J. I. \& Ruiz-Omeñaca, J. I. 2003. Presencia de un terópodo gigante en el Berriasiense inferior de las Villasecas (Soria, Cuenca de Cameros). Libro de Resúmenes. XIX Jornadas de la Sociedad Española de Paleontología. Morella 2003, 32.

Benton, M. J., Cook, E., Grigorescu, D., Popa, E. \& Tallódi, E. 1997. Dinosaurs and other tetrapods in an Early Cretaceous bauxite-filled fissure, northwestern Romania. Palaeogeography, Palaeoclimatology, Palaeoecology, 130, 275-292.

Bonde, N. \& Christiansen, P. 2003. New dinosaurs from Denmark. Comptes Rendus Palevol, 2, 13-26.

Buffetaut, E. \& Ouaja, M. 2002. A new specimen of Spinosaurus (Dinosauria, Theropoda) from the Lower Cretaceous of Tunisia, with remarks on the evolutionary history of the Spinosauridae. Bulletin de la Société géologique de France, 173, 415-421.

Calvo, J. O. 1991. Huellas de dinosaurios en la Formación Rio Limay (Albiano-Cenomaniano?), Picun Leufu, Provincia de Neuqen, República Argentina. (Ornithischia-Saurischia: Sauropoda-Theropoda). Ameghiniana, 28, 241-258.

Calvo, J. A. 1999. Dinosaurs and other vertebrates of the Lake Ezequiel Ramos Mexia area, Neuquén-Patagonia, Argentina. In: Proceedings of the Second Gondwanan Dinosaur Symposium (Eds. Y. Tomida, T.H. Rich \& P. Vickers-Rich). National Science Museum Monographs, 15, Tokyo, 13-45.

Calvo, J. O. \& Coria, R. 1998. New specimen of Giganotosaurus carolinii (Coria \& Salgado, 1995), supports it as the largest theropod ever found. In: Aspects of Theropod Paleobiology (Eds. B. P. Pérez Moreno, T. Holtz Jr., J. L. Sanz \& J. Moratalla). Gaia, 15, 117-122

Casanovas-Cladellas, M. L. \& Santafé-Llopis, J. V. 1971. Icnitas de reptiles mesozoicos en la provincia de Logroño. Acta Geológica Hispánica, 5, 139-142.

Casanovas, M. L. \& Santafé, J. V.1974. Dos nuevos yacimientos de icnitas de Dinosaurios. Acta geologica Hispanica, 9, 88-91.

Casanovas, M.L., Fernández, A., Pérez-Lorente, F. \& Santafé, J.V. 1995. Un terópodo carnosaurio en el camino a Treguajantes (La Rioja, España). In: Huellas fósiles de dinosaurios de La Rioja: nuevos yacimientos (Coord. F. Pérez-Lorente). Instituto de Estudios Riojanos (Ciencias de la Tierra, 18), Logroño, 13-14.

Casanovas, M.L., Fernández, A., Pérez-Lorente, F. \& Santafé, J.V. 1997. Sauropod trackways from site El Sobaquillo (Munilla, La Rioja, Spain) indicate amble walking. Ichnos, 5, 101-107.

Charig A. J. \& Milner, A. C. 1997. Baryonyx walkeri, a fisheating dinosaur from the Wealden of Surrey. Bulletin of the Natural History Museum of London (Geology Series), 53,11-70.

Christiansen, P. \& Bonde, N. 2003. The first dinosaur from Denmark. Neues Jahrbuch für Geologie und Paläontologie, Abhandlungen, 227, 287-299.

Chure, D. J. 2000. A new species of Allosaurus from the Mo- 
rrison Formation of Dinosaur National Monument (UT$\mathrm{CO}$ ) and a revision of the theropod Family Allosauridae. Tesis doctoral, Columbia University, 1-964 (inédita).

Coria, R. \& Salgado, L. 1995. A new giant carnivorous dinosaur from the Cretaceous of Patagonia. Nature, 377, 224-226.

Currie, P. J. 2003. Cranial anatomy of tyrannosaurid dinosaurs from the Late Cretaceous of Alberta, Canada. Acta Palaeontologica Polonica, 48, 191-226.

Currie, P. J. \& Carpenter, K. 2000. A new specimen of Acrocanthosaurus atokensis (Theropoda, Dinosauria) from the Lower Cretaceous Antlers Formation (Lower Cretaceous, Aptian) of Oklahoma, USA. Geodiversitas, 22, 207-246.

Currie, P. J., Hurum, J. H. \& Sabath, K. 2003. Skull structure and evolution in tyrannosaurid dinosaurs. Acta Palaeontologica Polonica, 48, 227-234.

Currie, P. J. \& Zhao X.-J., 1993. A new carnosaur (Dinosauria, Theropoda) from the Jurassic of Xinjiang, People's Republic of China. Canadian Journal of Earth Sciences, 30, 2037-2081.

Dames, W. 1884. Vorlegung eines Zhanes von Megalosaurus aus dem Wealden des Deisters. Sitzungsverichte der Gesellschaft Naturforschender Freunde zu Berlin, 1884, 186-188.

Farlow, J. O. 2001. Acrocanthosaurus and the maker of Comanchean large-theropod footprints. In: Mesozoic Vertebrate Life (Eds. D. H. Tanke \& K. Carpenter). Indiana University Press, Bloomington and Indianapolis, 408-427.

Fuentes Vidarte, C. 1996. Primeras huellas de aves en el Weald de Soria (España): Nuevo icnogénero Archaeornithipus y nueva icnoespecie A. meijidei. Estudios Geológicos, 52, 63-75.

Fuentes Vidarte, C. \& Meijide Calvo, M. 1998. Icnitas de dinosaurios terópodos en el Weald de Soria (España). Nuevo icnogénero Kalohipus. Estudios Geológicos, 54, 147-152.

Fuentes Vidarte, C., Meijide Calvo, M., Meijide Fuentes \& Huerta Hurtado, P. 2001. Las huellas de Dinosaurios de Castilla y León. In: Patrimonio Geológico de Castilla y León (Ed. R. Nuche del Rivero). Enresa, Madrid, 360379 .

Gabuniya, L. K. \& Kurbatov, V. V. 1982. Jurassic dinosaur tracks of Tashkurgan (Uzbekistan SSR). In: Abstracts of the scientific session, Tbilisi, 20-22.

Grigorescu, D. 2003. Dinosaurs of Romania. Comptes Rendus Palevol, 2, 97-101.

Gómez Fernández, J. C. \& Meléndez, N. 1994. Estratigrafía de la "Cuenca de los Cameros" (Cordillera Ibérica Noroccidental, $\mathrm{N}$ de España) durante el tránsito JurásicoCretácico. Revista de la Sociedad Geológica de España, 7, 121-139.

Harris, J. D. 1998. A reanalysis of Acrocanthosaurus atokensis, its phylogenetic status, and paleobiogeographic impliations, based on a new specimen from Texas. Bulletin of the New Mexico Museum of Natural History and Science, 13, 1-75.
Holtz, T. R., Jr. 1998. A new phylogeny of the carnivorous dinosaurs. In: Aspects of Theropod Paleobiology (Eds. B. P. Pérez Moreno, T. Holtz Jr., J. L. Sanz \& J. Moratalla). Gaia, 15, 5-61.

Hurum, J. H. \& Sabath, K. 2003. Giant theropod dinosaurs from Asia and North America: Skulls of Tarbosaurus bataar and Tyrannosaurus rex compared. Acta Palaeontologica Polonica, 48, 161-190.

Hutt, S., Martill, D. M. \& Barker, M. J. 1996. The first European allosaurid dinosaur (Lower Cretaceous, Wealden Group). Neues Jahrbuch für Geologie und Paläontologie, Monatshefte, 1996, 635-644.

Hutt, S., Naish, D., Martill, D. M., Barker, M. J. \& Newbery, P. 2001. A preliminary account of a new tyrannosauroid theropod from the Wessex Formation (Early Cretaceous) of southern England. Cretaceous Research, 22, $227-$ 242.

Jurcsák, T., 1982. Occurrences nouvelles des sauriens mésozoïques de Roumanie. Vertebrata Hungarica, 21, 175-184.

Jurcsák, T. \& Popa, E., 1983. La faune de dinosauriens du Bihor (Roumanie). In: Actes du Symposium Paléontologique Georges Cuvier, Montbéliard, France, 1982 (Eds. E. Buffetaut, J. M. Mazin \& E. Salmon). Montbéliard, 325-335.

Kellner, A.W.A. \& Campos, D. A. 1996. First early Cretaceous theropod dinosaur from Brazil, with comments on Spinosauridae. Neues Jahrbuch für Geologie und Paläontologie, Monatshefte, 1996, 151-166.

Langston, W. Jr. 1974. Nonmammalian Comanchean tetrapods. Geoscience and Man, 8, 77-102.

Lires, J. F., Piñuela, L. \& Garcia-Ramos, J. C. 2001. Nuevos datos y reinterpretación del yacimiento jurásico de icnitas de dinosaurios de la playa de La Griega (Colunga, Asturias). Publicaciones del Seminario de Paleontología de Zaragoza (SEPAZ), 5 (1), 342-347.

Lockley, M. 1998. Philosophical perspectives on theropod track morphology: blending qualities and quantities in the science of ichnology. In: Aspects of Theropod Paleobio$\operatorname{logy}$ (Eds. B. P. Pérez Moreno, T. Holtz Jr., J. L. Sanz \& J. Moratalla). Gaia, 15, 279-300.

Lockley, M. G. 2000. An amended description of the theropod footprint Bueckeburgichnus maximus Kunh, 1958, and its bearing on the megalosaur tracks debate. Ichnos, 7, 217-225

Lockley, M. G. \& Hunt, A. P. 1994. A track of the giant theropod dinosaur Tyrannosaurus from close to the Cretaceous/Tertiary Boundary, northern New Mexico. Ichnos, 3, 213-218.

Lockley, M. G., Meyer, C. A. \& Moratalla, J. J. 1998a. Therangospodus: trackway evidence for the widespread distribution of a late Jurassic theropod with well-padded feet. In: Aspects of Theropod Paleobiology (Eds. B. P. Pérez Moreno, T. Holtz Jr., J. L. Sanz \& J. Moratalla). Gaia, 15, 339-353.

Lockley, M. G., Meyer, C. A. \& Santos, V. F. dos. 1998b. Megalosauripus and the problematic concept of megalosaur footprints. In: Aspects of Theropod Paleobiology (Eds. B. 
P. Pérez Moreno, T. Holtz Jr., J. L. Sanz \& J. Moratalla). Gaia, 15, 313-337.

Lockley, M. G., Meyer, C. A., Schultz-Pittman, R. \& Forney, G. 1996. Late Jurassic Dinosaur tracksites from Central Asia: A preliminary report on the world's longest trackways. Museum of Northern Arizona Bulletin, 60 , 137-140.

Martín-Closas, C. \& Alonso Millán, A. 1998. Estratigrafía y bioestratigrafía (Charophyta) del Cretácico Inferior en el sector occidental de la Cuenca de Cameros (Cordillera Ibérica). Revista de la Sociedad Geológica de España, 11, 253-269.

Madsen, J. H., Jr. 1976. Allosaurus fragilis: A revised osteology. Utah Geological and Mineral Survey, Bulletin, 109, 1-163.

Mas, J. R., Alonso, A. \& Guimera, J. 1993. Evolución tectonosedimentaria de una cuenca extensional intraplaca: La cuenca finijurásica-eocretácica de Los Cameros (La Rioja-Soria). Revista de la Sociedad Geológica de España, 6, 129-144.

McCrea, R. T., Curre, P. \& Pemberton, S. 2002. Forelimb impressions associated with a large theropod trackway from the Gates Formation (Lower Cretaceous; Albian) of Western Canada. Journal of Vertebrate Paleontology, 22 (supplement to number 3), 86A.

Mensink, H. \& Mertmann, D. 1984. Dinosaurier-Fährten (Gigantosauropus asturensis n.g. n. sp.; Hispanosauropus hauboldi n.g. n. sp.) im Jura Asturiens bei La Griega und Ribadesella (Spanien). Neues Jahrbuch Geologie Paläontologie Monatshefte, 1984, 405-415.

Meyer, C. A. 2000. The Rio Limay vertebrate ichnofauna (Cretaceous, Patagonia) revisited - evidence for a Cenomanian not Albian age of Giganotosaurus. 5th European Workshop on Vertebrate Palaeontology, Karlsruhe. 27.06- 01.07.2000. Program. Abstracts. Excursion guides, 52-53.

Milner, A. 1997. Spinosauridae and Baronychidae. In: Encyclopedia of Dinosaurs (Eds. P. J. Currie \& K. Padian). Academic Press, San Diego, 699-700.

Milner, A. 2002. Theropod dinosaurs of the Purbeck limestone group, Southern England. In: Life and environments in Purbeck times (Eds. A. R. Milner \& D. J. Batten). Special Papers in Palaeontology, 68, 191-201.

Moratalla-García, J. J. 1993. Restos indirectos de dinosaurios del registro español: Paleoicnología de la Cuenca de Cameros (Jurásico superior-Cretácico inferior) y Paleoología del Cretácico superior. Tesis Doctoral, Universidad Complutense de Madrid, 1-727 (inédita).

Moratalla, J. J. \& Sanz, J. L. 1997. Cameros Basin megatracksite. In: Encyclopedia of Dinosaurs (Eds. P. J. Currie \& K. Padian). Academic Press, San Diego, 87-89.

Naish, D. 2002. The historical taxonomy of the Lower Cretaceous theropods (Dinosauria) Calamospondylus and Aristosuchus from the Isle of Wight. Proceedings of the Geologists'Association, 113, 153-163.

Naish, D., Hutt, S. \& Martill, D.M. 2001. Saurichian (sic) dinosaurs 2: Theropods. In: Dinosaurs of the Isle of Wight (Eds D.M. Martill \& D. Naish). The Paleontological Association. Field Guide to Fossils, 10, 242-309.
Osborn, H. F. 1924. Three new Theropoda Protoceratops Zone, central Mongolia. American Museum Novitates, 144, 1-12.

Owen, R. 1854. On some fossil reptilian and mammals remains from the Purbecks. Quaterly Journal of the Geological Society of London, 10, 420-433.

Pascual-Arribas, C. \& Sanz-Pérez, E. 2000. Icnitas de dinosaurios en Valdelavilla (Soria, España). Estudios Geológicos, 56, 41-61.

Paul, G. S. 1988. Predatory dinosaurs of the world. Simon and Schuster, New York, 1-464.

Pérez-Lorente, F. 2004. Icnitas de dinosaurios del Cretácico en España. In: Dinosaurios y otros Reptiles Mesozoicos de España. (Coord. F. Pérez-Lorente). Instituto de Estudios Riojanos (Ciencias de la Tierra, 26), Logroño, 49-108.

Pérez-Lorente, F., Romero-Molina, M. M., Requeta, E., Blanco, M. \& Caro, S. 2001. Dinosaurios. Introduccion y análisis de algunos yacimientos de sus huellas en La Rioja. Ciencias de la Tierra, 24, 1-102.

Pérez-Moreno, B.P., Chure, D.J., Pires, C., Marques da Silva, C., Dos Santos, V., Dantas, P., Povoas, L., Cachao, M., Sanz, J.L. \& Galopim de Carvalho, A. M. 1999. On the presence of Allosaurus fragilis (Theropoda: Carnosauria) in the Upper Jurassic of Portugal: first evidence of an intercontinental dinosaur species. Journal of the Geological Society of London, 156, 449-452.

Pittman, J. G. 1989. Stratigraphy, lithology, depositional environment, and track type of dinosaur track-bearing beds of the Gulf Coastal Plain. In: Dinosaur Tracks and Traces (Eds. D. D. Gillette \& M. G. Lockley). Cambridge University Press, Cambridge, New York, Port Chester, Melboune, Sydney, 135-153.

Pittman, J. G. 1992. Stratigraphy and Vertebrate ichnology of the Glen Rose Formation, Western Gulf Basin, USA. Tesis doctoral, University of Texas at Austin, 1-726 (inédita).

Platt, N. H. \& Meyer, C. A. 1991. Dinosaur footprints from the Lower Cretaceous of northern Spain: their sedimentological and palaeoecological context. Palaeogeography, Palaeoclimatology, Palaeoecology, 85, 321-333.

Rauhut, O. W. M. 2003. A tyrannosauroid dinosaur from the Upper Jurassic of Portugal. Palaeontology, 46, 903-910.

Rubio de Lucas, J. L. 2001. Tierra de Yanguas: guía interpretativa de los dinosaurios a través de las cañadas. Ed. Luzán 5, S. A. de ediciones, Madrid, 1-311.

Ruiz-Omeñaca, J. I. \& Canudo, J. I. 2001. Dos yacimientos excepcionales con vertebrados continentales del Barremiense (Cretácico Inferior) de Teruel: Vallipón y La Cantalera. Naturaleza Aragonesa, 8, 8-17.

Ruiz-Omeñaca, J. I. \& Canudo, J. I. 2003. Un nuevo dinosaurio terópodo ("Prodeinodon" sp.) en el Cretácico Inferior de La Cantalera (Teruel). Geogaceta, 34, 111-114.

Ruiz-Omeñaca, J. I., Canudo, J. I. \& Cuenca Bescós, G. 1998. Primera cita de dinosaurios barinonícidos (Saurischia: Theropoda) en el Barremiense superior (Cretácico Inferior) de Vallipón (Castellote, Teruel). Mas de las Matas, 17, 201-223.

Russell, D. A. 1996. Isolated dinosaur bones from the Middle Cretaceous of the Tafilalt, Morocco. Bulletin du Muséum National d'Histoire Naturelle, 4e série, Section C (Scien- 
ces de la Terre: Paléontologie, Minéralogie, Géologie), 18, 349-402.

Santos, V. F. dos, Carvalho, A. M. \& Silva, C. M. 1995. A jazida da Pedreira da Ribeira do Cavalo (Sesimbra) ou a história das pegadas de dinossáurios que nunca mais poderemos visitar. Al-madan, II Série, 4, 175-177.

Sanz, J. L, Moratalla, J. J. \& Casanovas, M. L. 1985. Traza icnológica de un Dinosaurio Iguanodóntido en el Cretácico inferior de Cornago (La Rioja, España). Estudios Geológicos, 41, 85-91.

Sanz, J. L., Moratalla, J. J., Rubio, J. L., Fuentes, C. \& Meijide, M. 1997. Huellas de dinosaurios de Castilla y León. Junta de Castilla y León, Burgos y Soria, 1-87.

Sanz, E., Pascual, C \& Bárez, S. 1999. Icnitas de dinosaurios en la facies Weald de "El Royo" (Soria, España). Boletín de la Real Sociedad Española de Historia Natural (Sección geológica), 95, 101-115.

Sereno, P. C., Beck, A.L., Dutheil, D. B., Gado, B., Larsson, C.E., Lyon, G. H., Marcot, J. D., Rauhut, O.W., Sadleir, R. W., Sidor, C. A., Varricchio, D. D., Wilson, G. P. \& Wilson, J.A. 1998. A long-snouted predatory dinosaur from Africa and the evolution of spinosaurids. Science, 282, 1298-1302.

Sereno, P. C., Dutheil, D. B., Iarochene, M., Larsson, H. C. E., Lyon, G. H., Magwene, P. M., Sidor, C. A, Varricchio, D. J. \& Wilson, J. A. 1996. Predatory dinosaurs from the Sahara and late Cretaceous faunal differentiation. Science, 272, 986-991.
Shuler, E. W. 1935. Dinosaur tracksmounted in the bandstand at Glen Rose, Texas. Field and Laboratory, 4, 9-13.

Smith, D. K. 1998. A morphometric analysis of Allosaurus. Journal of Vertebrate Paleontology, 18, 126-142.

Sternberg, C. M. 1932. Dinosaur tracks from Peace River, British Columbia. National Museum of Canada, Annual Report, 1930, 59-85.

Sues, H.-D., Frey, E., Martill, D.M. \& Scott, D.M. 2002. Irritator challengeri, a spinosaurid (Dinosauria: Theropoda) from the Lower Cretaceous of Brasil. Journal of Vertebrate Paleontology, 22, 535-547.

Thulborn, T. 1990. Dinosaur tracks. Chapman and Hall, London, New York, Tokyo, Melbourne, Madras, 1-410.

Valenzuela, M., García-Ramos, J. C. \& Suarez de Centi, C. 1986. Los dinosaurios de Asturias a partir de sus huellas. Asturia, 5, 66-71.

Valenzuela, A.M., García-Ramos, J.C. \& Suarez de Centi, C. 1988. Las huellas de dinosaurios del entorno de Ribadesella. Central Lechera Asturiana, Oviedo, 1-34.

Wright, J. L., Barrett, P. M., Lockley, M. G. \& Cook, E. 1998. A review of the Early Cretaceous Terrestrial Vertebrate Track-Bearing Strata of England and Spain. Lower and Middle Cretaceous Terrestrial Ecosystems. New Mexico Museum of Natural History and Science, 14,143-153.

Manuscrito recibido: 16 de Febrero, 2004 Manuscrito aceptado: 19 de Abril, 2005 